\title{
A new classification of glaucomas
}

\author{
Constantin-Dan Bordeianu \\ Private Practice, Ploiesti, Prahova, \\ Romania
}

This article was published in the following Dove Press journal:

Clinical Ophthalmology

II September 2014

Number of times this article has been viewed

Purpose: To suggest a new glaucoma classification that is pathogenic, etiologic, and clinical.

Methods: After discussing the logical pathway used in criteria selection, the paper presents the new classification and compares it with the classification currently in use, that is, the one issued by the European Glaucoma Society in 2008.

Results: The paper proves that the new classification is clear (being based on a coherent and consistently followed set of criteria), is comprehensive (framing all forms of glaucoma), and helps in understanding the sickness understanding (in that it uses a logical framing system). The great advantage is that it facilitates therapeutic decision making in that it offers direct therapeutic suggestions and avoids errors leading to disasters. Moreover, the scheme remains open to any new development.

Conclusion: The suggested classification is a pathogenic, etiologic, and clinical classification that fulfills the conditions of an ideal classification. The suggested classification is the first classification in which the main criterion is consistently used for the first 5 to 7 crossings until its differentiation capabilities are exhausted. Then, secondary criteria (etiologic and clinical) pick up the relay until each form finds its logical place in the scheme. In order to avoid unclear aspects, the genetic criterion is no longer used, being replaced by age, one of the clinical criteria. The suggested classification brings only benefits to all categories of ophthalmologists: the beginners will have a tool to better understand the sickness and to ease their decision making, whereas the experienced doctors will have their practice simplified. For all doctors, errors leading to therapeutic disasters will be less likely to happen. Finally, researchers will have the object of their work gathered in the group of glaucoma with unknown or uncertain pathogenesis, whereas the results of their work will easily find a logical place in the scheme, as the suggested classification remains open to any new development.

Keywords: glaucoma, classification of glaucoma, pathogenic forms of glaucoma

\section{Introduction}

The main criticism of the existing glaucoma $(\mathrm{G})$ classifications, including the classification issued by the European Glaucoma Society in 2008 (2008 EGSc), ${ }^{1}$ concerns the criteria on which the whole construction is built.

In my opinion, an ideal classification should have the following characteristics: 1) all criteria should be beyond criticism (sooner or later, a classification based on criticizable criteria will be abandoned); 2) each crossing should be based on one criterion only, because too many criteria used at the same time for the same crossing may confuse not only the young and inexperienced doctor but also the experienced one, and may favor abandonment (I am using the term "crossing", because I perceive the classification as a map with several successive crossings, on which the doctor, guided by every piece of available information, is able to find the right place for every new case, in such a way that the therapeutic decision is eased: the alternate term is "divisions"); 3) each clinical form should be placed in one single locus; and 4) a specific treatment should correspond to each of the described categories.
Correspondence: Constantin-Dan Bordeianu

I5, Cameliei St, BI 26, Sc B, Et I, Ap 26

Ploiesti, I0009I, Prahova, Romania

Tel +40 2445989 I5; +40 720328040

$\mathrm{Fax}+40244598915$

Email bordmail3@yahoo.com 
When we refer to the fundamental criterion, it should be all-inclusive, valid for all described forms of sickness and leaving space even for forms of sickness that have not yet been described. Moreover, it should be consistently used in several successive crossings until its differentiation capabilities are exhausted. From that moment on, a secondary criterion must clarify the resulting categories until its capabilities are in turn exhausted and so on until each clinical form finds its natural place.

If we refer to the secondary criteria, they should be used in such a manner that every aspect of the sickness is covered. These criteria must deepen the differentiation capabilities, going from general to particular, progressively narrowing the comprehension sphere in a logical manner. Moreover, each secondary criterion must be applicable at any particular crossing to all categories already described at the previous level of analysis. This means that the criteria used to differentiate the members of one category must be valid for the cases entering its twin category/ies: the criteria used for the primary forms should be valid for the secondary forms, as well. Finally, the "one criterion for each form" principle may be used only at the end, when the clinical entities will be individualized (see the section "The third secondary criterion" under "The choice of secondary criteria").

If all these rules are respected, the resulting G classification will be clear - based on a coherent and constantly followed set of criteria; comprehensive, hosting all already described forms; and open, offering a place even for forms that have not yet been described. This classification will help in understanding the sickness by framing all its forms in a logical system. Finally, it will ease therapeutic decision making: guided by such a classification in which a specific treatment is described for each category, even if the reader takes a longer time to reach the end of his thinking pathway, his efforts will be rewarded by the ease with which the correct treatment is found.

On the contrary, in any of the existing G classifications (the 2008 EGSc included), after placing the case in one gonioscopic category, the user will be forced to the new level of pathogenic analysis to find the correct treatment. Reaching the end of the thinking pathway imposed by these classifications without finding a practical solution, the user might consider that all his previous framing efforts were useless. Being unable to understand why the authors have used criteria that cannot make enough difference by themselves in a construction that is unable to ease the therapeutic choice, the user might be tempted to abandon the classification that did not provide enough help. This may explain why terms like "chronic simple G" and “congestive G," which are specific to Donders' classification, ${ }^{2}$ are still in use. The purpose of this paper is to describe a new classification of glaucoma, intended to maintain the only advantage of the 2008 EGSc and to annul its shortcomings, which are listed in Table 1 and discussed in detail in my previous paper. $^{3}$

\section{Materials and methods}

\section{The choice of the fundamental criterion}

In my previous paper, ${ }^{3}$ I proved that both the gonioscopic criterion (distinguishing the open angle $\mathrm{G}$ and the angle closure G), both the etiologic criterion (distinguishing the primary $\mathrm{G}$ and the secondary $\mathrm{G}$ ) and the genetic criterion (distinguishing the congenital $\mathrm{G}$ and the acquired $\mathrm{G}$ ), are not beyond criticism and cannot suggest a specific treatment for each particular case. As consequence, the $\mathrm{G}$ classifications based on these criteria cannot have practical finality: they cannot ease the decision making by grouping the forms in such categories that a specific treatment would correspond to each category. In my opinion, only a classification using the pathogenic criterion as the fundamental one is capable of accomplishing this purpose, because currently the pathogenic mechanism is known for most forms, so that the best we can do is to conduct the treatment after the results of a pathogenic analysis, attacking the essential link in the pathogenic chain.

In the attempt to suggest a new pathogenic classification, I will not adopt the manner in which Allingham et al ${ }^{4}$ tried to group the $\mathrm{G}$ forms according to the pathogenic mechanism. In fact, Allingham's classification remains a gonioscopic one, and almost every shortcoming listed in Table 1 remains valid. I also will not use the way in which Ourgaud and Etienne ${ }^{5}$ and Krasnov ${ }^{6}$ used the categories described by Goldmann: ${ }^{7}$ these authors focused only on the part of the morbid process connected with the cilio-trabeculo-episcleral segment of the eye, neglecting everything that happens at the level of the optic nerve head because of local or general conditions. Even without this omission, these pathogenic classifications remain inoperative because from the three described categories (hypersecretion G, G with increased flow resistance, and G with increased episcleral vein pressure), the first and third forms are rare or extremely rare: that is why these classifications have not withstood time. Nevertheless, these categories will be used in my classification but placed in a more detailed scheme $^{8-10}$ for the purpose of clarifying the sickness by following in a logical manner its pathogenic pathway. In this scheme, each crossing will separate one category that needs to be further detailed and one to two other categories that do not need further analysis, or need less analysis at the moment. 


\section{The main pathogenic crossings}

The first pathogenic crossing (see Figure 1) will describe the "known pathogeny G" and the "unknown or uncertain pathogeny G." The last category seems useless because the pathogenesis of most acquired forms is known. Nevertheless, we must consider the future, when new forms might be described. We must think of many of the forms framed today as congenital glaucomas (Gs). Because of their rareness, some of these forms have an obscure pathogenesis and cannot find a locus of their own in a pathogenic classification. Until their pathogenesis is clarified, these forms will be gathered in the group of unknown or uncertain pathogeny $\mathrm{G}$.

The second pathogenic crossing will detail the known pathogeny G, separating the "simple pathogeny G" and the "complex pathogeny G": I am aware that almost every form may have a complex pathogeny in which a secondary mechanism or mechanisms may modulate the effect of the main one. Nevertheless, to analyze the sickness from the pathogenic point of view, we must assume that simple cases do exist. We will come back later to the complex pathogeny forms because the complexity may exist at different levels, which must first be delineated.
Keeping this in mind, the third pathogenic crossing will divide the simple pathogeny Gs in "pressure aggression G" (PAG) and "tissue resistance alteration G" (TRAG). I suggested the last term starting from the idea that the visual structures are characterized by an individual level of tissue resistance in front of aggressions, a level that may be normal or altered. The term TRAG clearly defines the group previously named "low (or normal) pressure G," a criticizable term from many points of view. First, it is not the low intraocular pressure (IOP) that determines the anatomic and functional alterations specific for $\mathrm{G}$, but the tissue resistance in front of pressure aggression is low and does not resist even at IOP levels within the normal statistical range: in some cases, it is not certain that the IOP plays any role. ${ }^{4,11,12}$ Second, the term "low pressure G" is applicable only to the cases with simple pathogeny, low pressure, and open angle: in reality, the tissue resistance alteration may intervene in any form of $\mathrm{G}$, modulating the effects of pressure aggression. Third, the term "low pressure G" draws the attention toward the rapport between the production and the evacuation of aqueous humor. It narrows the thinking sphere because the real mechanism acts at the level of optic neural tissue. Unfortunately, none of these aspects is reflected by the term "low pressure G."

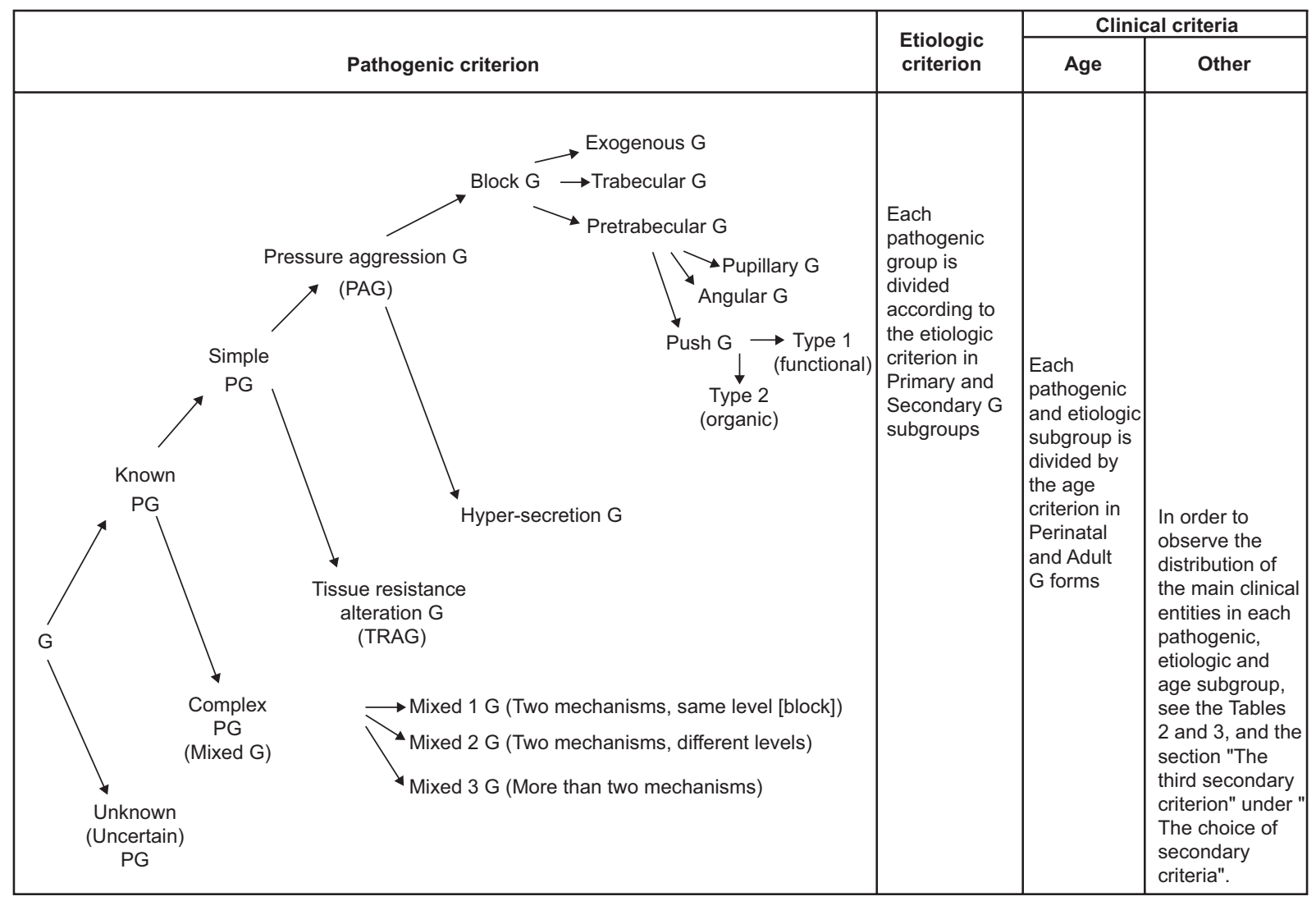

Figure I The pathogenic classification of glaucomas.

Abbreviations: G, glaucoma; PAG, pressure aggression glaucoma; PG, pathogeny glaucoma; TRAG, tissue resistance alteration glaucoma. 
On the contrary, the term "TRAG" points toward the real mechanism and broadens the thinking sphere. This term has proved its usefulness since its suggestion: ${ }^{8}$ it has been able to host a wide variety of influences (IOP, vascular, circulatory, neurological, metabolic, genetic, etc), some of which had not yet been described in 1986. At that time, extremely few spoke about neuroprotection and all its implications in G. TRAG is a term that has suggested neuroprotection since that period, before neuroprotection became generally accepted. For the present day, the main benefit of the suggested dichotomy (PAG-TRAG) consists in the fact that TRAG gets the right of independent existence, not only as a form of primary open angle G (POAG). As consequence, we are allowed to think that tissue resistance alteration (TRA) may influence the evolution of any type of $\mathrm{G}$ and we are allowed to think of neuroprotection in any type of G. For the future, the term is generous, too, because it leaves space for any new development. For example, it suggests the possibility of tissue resistance increase (TRI), which may act as a protective agent.

The fourth pathogenic crossing will divide the pressure aggression Gs in "block G" and "hypersecretion G." The "block $G$ " is the usual form, while hypersecretion has been included in the scheme only to cover a theoretical possibility, when hypersecretion may act as the unique mechanism. Usually it acts as a complementary phenomenon in the genesis of secondary OA uveitic Gs (Posner-Schlossman syndrome included), caused by a breakdown of the blood-aqueous barrier.

The fifth pathogenic crossing describes the following forms of block G: "exogenous (block) G," "trabecular (block) G," and "pretrabecular (block) G." For conciseness, we may omit the word "block," which is implied. As consequence, the three forms of block G are: "exogenous G," "trabecular G," and "pretrabecular G." It is clear that from hereon we walk on firm ground because these forms do exist in any gonioscopic classification currently in use: exogenous $\mathrm{G}$, open-angle $\mathrm{G}(\mathrm{OAG})$, and angle-closure $\mathrm{G}(\mathrm{ACG})$. Besides the new pathogenic names for OAG and ACG, the essential difference consists in the fact that exogenous $G$ gets the right of independent existence, instead of being crowded against logic into the group of OAG. As consequence, the situations of logic fracture listed in Table 1, line C-V-3 and discussed in detail in my previous paper, ${ }^{3}$ no longer appear.

The sixth pathogenic crossing differentiates the pretrabecular $\mathrm{G}$ according to the site where the pathogenic first movement happens, because the pretrabecular block may be produced by different mechanisms responding to different, sometimes opposite, therapeutic gestures. The "pupillary (block) G" is defined as occurring when the angle is closed by a factor acting in the pupil, the "angular (block) G" is defined as occurring when the angle is closed by a factor acting in the angle, while the "(posterior) push G" is defined as occurring when the causative factor acts behind the lens-zonule plane. For conciseness, we may omit the words "block" and "posterior," which are implied. As consequence, the three forms of pretrabecular G are "pupillary G," "angular G," and "push G".

The seventh pathogenic crossing clarifies the group of push G. It separates the push $1 \mathrm{G}$ (functional push $\mathrm{G}$ ) which results from functional disorders (aqueous misdirection to an intra-retrovitreal or supraciliary space; uveal effusion syndrome), and the push $2 \mathrm{G}$ (organic push $\mathrm{G}$ ), a rarer form, which results from organic alterations (detailed in Tables 2 and 3 and the section "The third secondary criterion").

\section{The complex pathogeny Gs (mixed Gs)}

These forms are determined by two or more mechanisms.

The mixed $1 \mathrm{G}$ associates two mechanisms situated at the same level (level 6: mechanisms leading to block), and the following combinations may be described: exogenous and trabecular, trabecular and pretrabecular, and pretrabecular and exogenous. Any form of pretrabecular block may be associated, but usually the pupillary block is involved. From multiple variants, the form that associates the trabecular and the pupillary block has already entered common knowledge in spite of the fact that it was not accepted by the 2008 EGSc. Another association neglected by the 2008 EGSc may appear in advanced G: any form of block $\mathrm{G}$ showing high levels of chronic eye hypertonia may develop a final stage of neovascu$\operatorname{lar} \mathrm{G}$, in which the vasoformative stimulus is generated by the retinal ischemia produced by the chronic high IOP itself.

The mixed $2 \mathrm{G}$ combines two mechanisms situated at different levels (see Figure 1): block (exogenous, trabecular, or pretrabecular) with tissue resistance (TR) abnormality, or with hypersecretion. Although it is not recognized as such, the combination block-TR abnormality is the most frequent manifestation of complex forms, because the effects of pressure aggression are always modulated by TR in front of pressure aggression. Usually, we find the block associated with TR alteration (TRA), but some cases might associate the block with TR increase (TRI). The mixed 3 Gs associate more than two mechanisms: any of the examples listed among the mixed 1 Gs may become complicated by TRA or TRI.

\section{Terminology aspects}

From the terminological point of view, new pathogenic names have been suggested for the simple pathogeny Gs. As for the cases with complex pathogeny, I think that the 
best way to give a name to each complex form is to respect the order in which the mechanisms intervened over time, noting the first active mechanism on which the second one has superimposed or is capable of superimposing. The name will indicate the group of mixed $\mathrm{G}$ by means of the connecting word interposed between mechanisms. When only a risk of association exists, the connecting word is the preposition "on" (for example, "trabecular, on narrow angle G." Other possible variants may be defined by replacing the first term with "exogenous," or with "TRAG," and the second term with "plateau iris configuration"). When both mechanisms are active, and produce mixed $1 \mathrm{G}$, the conjunction "and" is used (chronic pupillary and trabecular G; trabecular and pupillary G; exogenous and phacomorphic pupillary G; chronic pupillary and exogenous G; exogenous and push $1 \mathrm{G})$. When both mechanisms are active, and produce mixed 2 $\mathrm{G}$, the preposition "with" is used (trabecular G with TRA; trabecular G with TRI; TRA with trabecular G; other forms may be obtained by replacing the term "trabecular" with the term defining other types of pressure aggression: "exogenous", "pupillary", "angular", or "push"). When both mechanisms are active, and produce mixed $3 \mathrm{G}$, both connecting words are used (TRA with chronic pupillary and trabecular G; trabecular and pupillary in intercrisis G with TRA; exogenous with TRA and phacomorphic pupillary $\mathrm{G}$, etc).

The final aspect of the resulting pathogenic classification is shown in the first segment of Figure 1, under the heading "Pathogenic criterion". At the end of seven pathogenic crossings, nine pathogenic forms were identified, and for each form, a specific treatment exists: exogenous $G$, trabecular $G$, pupillary $\mathrm{G}$, angular $\mathrm{G}$, push $1 \mathrm{G}$, push $2 \mathrm{G}$, hypersecretion G, TRAG, and mixed G. With this, the role of the pathogenic criterion as the fundamental one has been accomplished. From hereon, secondary criteria must intervene to organize the clinical entities entering each group.

\section{The choice of the secondary criteria}

The choice of the secondary criteria and the order in which they will be used is of utmost importance for a classification to be clear and all-inclusive. The literature offers several suggestions: gonioscopic criterion, genetic criterion, etiologic criterion, age, evolution speed, complaint intensity, IOP level, associated congestion, compensation degree, treatment response, etc. Which one should be used? Which one should be used first?

\section{The first secondary criterion}

The first secondary criterion cannot be the gonioscopic one, and the reasons are abundantly offered in my previous paper. ${ }^{3}$
The genetic criterion cannot fulfill this role either, because it represents merely a birth-related criterion rather than a really genetic one: not every case of what is now named congenital $\mathrm{G}$ has genetic determinism, manifesting itself into families and respecting Mendelian laws (nevertheless, being generally accepted, I will maintain the term "genetic criterion"). Moreover, this criterion separates only a rare form of less than $1 \%$ with immature angle at birth from the rest of cases: the practical benefit is small. The clinical criterion also cannot fulfill the role of the first secondary criterion because none of the clinical signs have significance in every case. These signs will be used at the end, when a locus for each clinical entity must be created.

In my opinion, only the etiologic criterion is able to continue the differentiation activity at a superior level. According to this criterion, any pathogenic form of $\mathrm{G}$ may be primary (when no cause can be identified) or secondary (when the cause has been identified). This differentiation brings significant consequences, in spite of the fact that till now some rare forms are known to exist only as secondary manifestations (exogenous $\mathrm{G}$, push $2 \mathrm{G}$ ).

On further consideration, I think that the etiologic criterion covers a great part of what is now named genetic criterion: I think that in most primary acquired forms, the predisposition to develop glaucoma exists since birth either as a genetically transmitted trait or as a developmental anomaly that did not enter the genetic luggage. Thus, in primary pretrabecular $\mathrm{G}$, the angle narrowness and configuration (plateau iris) are genetically transmitted. ${ }^{11-13}$ In TRAG, genetic mutations have been described, ${ }^{14}$ and even families showing what is now called "normal tension G" have been identified. ${ }^{15}$ As for the primary trabecular G, although its heredity is not contested, ${ }^{16}$ the intimate mechanism leading to resistance increase is not known. ${ }^{4,11,12}$ In the absence of proved explanations, I believe that everything might be reduced to a genetically determined failure in the trabecular self-cleaning system. As long as this system functions, the debris that tends to clog the trabecular pores are captured by trabeculocytes and processed: the soluble part is poured into the aqueous humor and evacuated through the existing drainage pathways, whereas the insoluble part is transferred into the trabecular matrix through the basal membrane. I believe that the deficiency lies here: either a system that further transports the insoluble part does exist and this system fails with time, or such a system has not been created by nature, and everything depends on age, the functional capability of trabeculocytes, the abundance of debris an eye can produce, and the weight of the insoluble part in these debris. The last 
three characteristics are genetically determined, together with other individual characteristics. As a result of the accumulation of the insoluble part, the trabecular meshwork thickens and the pores diminish in caliber. Either of these assumptions would be true, what remains is that the trabeculum has an inborn validity period for its good function, a period that is genetically determined. Extending the idea, I think that the congenital $\mathrm{G}$ has a practically zero validity period, as the angle is not fully developed at birth.

The morphological and biochemical changes of the trabeculum in trabecular glaucoma are compatible with this line of thinking. On one hand, the basement membrane is thickened, suggesting increased cellular activity, ${ }^{17}$ and the endothelial cells number is decreased, ${ }^{18}$ possibly as a result of increased cell activity with subsequent wear. On the other hand, plaques made of clusters of material with unknown origin are accumulated in the extracellular matrix of the corneoscleral beams and juxtacanalicular meshwork. ${ }^{19}$ Other researchers have found quantitative and qualitative alterations of the extracellular matrix components. ${ }^{17,20-22}$

Summarizing all these findings and considerations, I think that most - if not all - primary Gs appearing in adults have congenital origins and cannot be considered as acquired, in the manner found in any existing classification, where the genetic criterion separates the cases into two opposite and mutually exclusive categories: congenital and acquired Gs. These classifications have paid too much attention to the physical aspect of the angle, neglecting its functional capabilities: the fully developed angle at birth - mark of the acquired $\mathrm{G}$ - may hide functional disabilities or predispositions with congenital origin, which may become clinically manifested later in life under the form of acquired G. If the existence of "late" congenital G is generally accepted, then I suggest that the forms considered till now to be primary acquired Gs be considered congenital anomalies with very late pathologic manifestation. In these conditions, if "primary" denotes "with congenital origin," then the use of etiologic and genetic criteria in the same classification becomes more harmful than helpful. Their meaning spheres overlap and generate many of the shortcomings listed in Table 1 (lines C-I-1, C-IV-2, C-IV-3, C-IX-1, and C-IX-2-a) and discussed in detail in my previous paper. ${ }^{3}$ To avoid these shortcomings, the etiologic criterion will be used alone in my classification, as the first secondary criterion, differentiating between primary forms (with congenital origin and - in adults - with very late pathologic manifestation) and secondary forms (the really acquired forms).

\section{The second secondary criterion}

In the classification suggested 27 years ago, ${ }^{8-10}$ this role was fulfilled by the genetic criterion. At that time, the idea that an immature angle at birth would produce pressure effects was widely accepted. As a consequence, I believed that the separation between cases with mature (acquired Gs) and immature angle at birth (congenital Gs) was important. Today, the situation has changed. Clinical experience has proved that some acquired cases could manifest themselves as congenital Gs if the IOP increases before 2 years of age. Other cases showing an immature angle at birth will not develop congenital G. This means that the differences in clinical manifestation, which result in differences in therapeutic attitude, depend more on the age at which the IOP begins to increase than on the presence of signs of insufficient angle differentiation. To additionally complicate the situation, most cases belonging to the former group of primary acquired $\mathrm{G}$ might be congenital anomalies or predispositions with very late pathologic manifestation, as discussed earlier.

For all these reasons, I believe that the genetic criterion with its congenital-acquired dichotomy can no longer be used as the second secondary criterion in a classification that is intended to be clear. In my opinion, the only criterion able to continue the differentiation activity at a superior level is age. According to this clinical criterion, G may be "perinatal" (up to 2 years of age, when the sickness associates buphthalmia or will associate it in a short period of time), or "adult" (after 2 years of age, when buphthalmia will never appear). The term "primary perinatal G" usually indicates a buphthalmic eye when no identifiable cause might be associated. The term "secondary perinatal G" means that an identifiable cause has prevented the complete development of the angle (G secondary to maternal rubella), altered a fully developed angle, or caused an increase in the episcleral vein pressure. The rest of the cases are adult-type, which does not need to be indicated in the name of the form. When there is no indication referring to age, it is presumed that $\mathrm{G}$ is adult-type, that is, the term "primary trabecular G" refers to the adult form of G.

Regarding the form previously described as "infantile G," one may observe that significant differences exist between the congenital and the infantile G, whereas the differences between the infantile and the adult form of trabecular $\mathrm{G}$ consist only of nuances. In fact, infantile $G$ has all the signs of an adult trabecular $\mathrm{G}$ but shows a poor surgical prognosis. This form is described in children and adolescents more often in Caucasian populations. Nevertheless, in black populations, almost every case suffering from adult trabecular $\mathrm{G}$ has poor surgical prognosis. As a consequence, I believe it is not worth 
Table I The advantage and the shortcomings of the 2008 EGSc compared with previous classifications

A. The 2008 EGSc as one step ahead of the previous classifications $(272,2,6)^{*}$

B. The 2008 EGSc as two steps behind the previous classifications $(273,2,3)$

I) The "OA-AC" dichotomy has disappeared from congenital $G(273,2,5)$

2) The secondary forms have disappeared from congenital $G(274, I, 3)$

C. The 2008 EGSc as similar to other previous classifications $(274,2,1)$

I. The 2008 EGSc uses criticizable fundamental criteria $(274,2,3)$

I) The genetic criterion (congenital-acquired) is criticizable because

a. The congenital-acquired dichotomy may not be real $(274,2,5)$

b. The use of congenital-acquired dichotomy may generate more troubles than benefits $(274,2,6)$

2) The etiologic criterion is criticizable because any $G$ is secondary to a cause $(274,2,7)$

3) The gonioscopic criterion is criticizable because

a. The angle aspect is only a clinical sign... $(274,2,8)$

b. The gonioscopic criterion cannot cover all cases... $(275, \mathrm{I}, \mathrm{I})$

c. Some statements in Terminology and Guidelines for Glaucoma' may confuse young opthtalmologists

(x) In secondary OAG, the angle may be closed at $90^{\circ}(275,1,7)$

(y) The same mechanism may lead to both OAG and ACG $(275,1,8)$

(z) Only an AC of more than $180^{\circ}$ needs prophylactic treatment $(275,2,2)$

II. The 2008 EGSc uses criticizable secondary criteria

I) The use of the associated congenital pathology in congenital G classification $(276,1,3)$

2) The use of etiologic agents as pathophysiologic mechanisms in secondary OAG $(276,1,5)$

3) The use of clinical stages only for PACG differentiation $(276, I, 6)$

4) The use of the pathogenic criterion only for secondary ACG differentiation $(276,2,2)$

III. The 2008 EGSc uses several criteria for one single crossing

I) The use of the genetic, etiologic, and gonioscopic criteria at the first crossing $(276,2,5)$

2) The use of age, IOP level, and lack of enough symptoms for POAG differentiation $(276,2,5)$

IV. The 2008 EGSc is not consistent in using a criterion $(277, \mathrm{I}, 4)$

I) The gonioscopic and etiologic criteria used for acquired $G$ are not used for congenital $G(277,1,4)$

2) The associated pathology used for congenital $G$ is not used for acquired $G(277,1,5)$

3) The age is used for acquired $G$ but is not used for congenital $G(277,1,6)$

4) The pathogenic criterion used for some acquired $A C G$ is not used for congenital $G(277,1,7)$

5) The pathogenic criterion is used only for secondary ACG, not for primary ACG $(277,2,2)$

V. The 2008 EGSc denies reality in some aspects

I) Associating tissue resistance alteration only with POAG $(277,2,4)$

2) Associating tissue resistance alteration only with low pressure $(277,2,5)$

3) Associating exogenous $G$ only with $\mathrm{OA}(277,2,6)$

4) Placing all stages of neovascular $G$ in the group of secondary ACG with pulling $(278, I, 2)$

5) Mingling the plateau iris $G$ with other PACG $(278,1,3)$

VI. The 2008 EGSc frames a sickness in more than one category $(278$, I, 4)

VII. The 2008 EGSc does not allow the clear framing of all forms of sickness

I) Glaucoma alterations + high IOP produced by trabecular obstruction on a narrow angle $(278,2,6)$

2) "POAG and narrow angle" presented in the hospital in a full AC attack in one eye $(278,2,7)$

3) Residual $G$ after opening of an angle that has remained closed for a long time $(279$, I, I)

4) Cases with hypertensive POAG showing different response to similar levels of IOP $(279,1,2)$

5) Cases with PACG showing different response to similar levels of IOP $(279$, I, 4)

6) All the mixed $G$ forms $(279,2,4)$

VIII. The 2008 EGSc does not offer direct therapeutical suggestions (280, I, 2)

IX. Miscellanea that might confuse young ophthalmologists

I) The absence of a definition at the beginning of any new chapter $(280,2,3)$

2) The absence of "clear mind" character from the editorial board

a. Terminologic confusions when naming the two categories of congenital G $(28 \mathrm{I}, \mathrm{I}, 3)$

b. Pathogenic confusions when identifying the PACG mechanisms $(28 \mathrm{I}, \mathrm{I}, 4)$

c. Errors in using the described AC mechanisms $(28 \mathrm{I}, 2,2)$

Notes: *At the end of each line in the outline in parentheses are the page number, column number, and paragraph number from Bordeianu ${ }^{3}$ where each characteristic is fully discussed. For example, $(272,2,6)$ refers to page 272 , column 2, paragraph 6 of Bordeianu. ${ }^{3}$

Abbreviations: AC, angle closure; ACG, angle closure glaucoma; 2008 EGSc, the classification issued by the European Glaucoma Society in 2008; G, glaucoma; IOP, intraocular pressure; OA, open angle; OAG, open angle glaucoma; PACG, primary angle closure glaucoma; POAG, primary open angle glaucoma. 
complicating the classification with infantile $\mathrm{G}$ because this type of response to treatment may be observed in adults, too. All one needs to know is that there are subjects in whom $G$ may relapse soon after surgery (young populations and black populations): on this basis, one can react accordingly.

\section{The third secondary criterion}

The third secondary criterion is represented by the remaining clinical signs, because at this point it is time to create a locus for each clinical entity. For this purpose, the "one criterion for each form" principle may be used, because frequently, one single significant clinical sign is enough to differentiate a clinical form (pigmentary G, pseudoexfoliation G, plateau iris $\mathrm{G}$, etc).

Using all the available information obtained from personal and familial pathologic history, from eye examination (biomicroscopy, gonioscopy, ultrabiomicroscopy [UBM], optical coherence tomography, tonometry, campimetry, perimetry, etc), from general and from laboratory examinations, and keeping in mind that only the forms appearing up to 2 years of age must contain the term referring to age in the name of the group, the clinical stage of the suggested classification reveals the following forms.

The primary adult $\mathrm{G}$ usually shows one clinical manifestation for each pathogenic mechanism, while the secondary adult $\mathrm{G}$ shows multiple forms for each of the pathogenic mechanisms (Table 2). Beside the data in this table, I will add a few words about the neovascular $\mathrm{G}$, because the 2008 EGSc has described only the common form, in which the retinal ischemia is secondary to organic vascular alterations (diabetic, atherosclerotic, etc), and in which G surgery must be preceded by the inhibition of vascular endothelial growth factor (VEGF): for details, see the section "The secondary forms" under "The new classification offers direct therapeutic suggestions". The 2008 EGSc has completely forgotten the form of neovascular $\mathrm{G}$ appearing in terminal stages of any form of block $\mathrm{G}$ manifested by chronic high levels of IOP: in this form, the retinal ischemia is secondary to the compression of relatively normal vessels by the chronic high IOP itself. It may be cured by G surgery alone, without any previous anti-VEGF therapy, because lowering the IOP will inhibit the angiogenetic factor release. In order to differentiate these

Table 2 The repartition of clinical entities in the pathogenic forms of adult G

\begin{tabular}{|c|c|}
\hline Pathogenic form & Clinical entities \\
\hline \multicolumn{2}{|l|}{ Primary adult G } \\
\hline - Trabecular G & former POAG in gonioscopic classification \\
\hline - Pupillary G & former PACG in gonioscopic classification \\
\hline - Angular G & plateau iris G \\
\hline - Push I G & rare cases of primary malignant $\mathrm{G}$ \\
\hline- TRAG & cases showing progressive G alterations and statistically normal IOP in the absence of any identifiable cause. \\
\hline \multicolumn{2}{|l|}{ Secondary adult $\mathbf{G}$} \\
\hline - Exogenous G & $\begin{array}{l}\text { G in case of chemical (alkali) or radiational damage of the episcleral veins, in Sturge Webber syndrome, in dural } \\
\text { shunts carotid-cavernous fistula, cavernous sinus thrombosis, superior vena cava obstruction, pulmonary venous } \\
\text { obstruction, retrobulbar or mediastinal tumors, endocrine ophthalmopathy, and in orbital phlebitis; }\end{array}$ \\
\hline - Trabecular G & $\begin{array}{l}\text { the obstruction agent determines the form: blood and altered macrophages (hemorrhagic G, ghost-cell G, } \\
\text { hemolytic G); lens material and altered macrophages (phakolytic G), pigment (pigmentary G); exfoliation material } \\
\text { (exfoliative G); inflammation with trabeculitis (uveitic G, G in scleritis, or episcleritis, G in posterior lens luxation, } \\
\text { G in case of silicone oil emulsion in anterior chamber, component in case of G in alkali burns); foreign agents } \\
\text { (OVDs); neoplastic cells and altered macrophages (besides the obstruction mechanism, the tumor may act by } \\
\text { direct compression or invasion). In traumatic G, besides the obstruction with blood, debris, or lens material, the } \\
\text { trabeculum may be broken and scarred (angle recession G). The corticosteroid induced G has a more complex } \\
\text { mechanism, but fits in the same group; }\end{array}$ \\
\hline - Pupillary G & $\begin{array}{l}\text { phakomorphic G, uveitic G (with pupil seclusion-occlusion), aphakic G (vitreous mushroom), pseudophakic G } \\
\text { (posterior synechia, pupil capture, capsular bag hydrops), inverse block pupillary G (anterior lens luxation, air or } \\
\text { other gas, or silicone oil into the anterior chamber); }\end{array}$ \\
\hline - Angular G & $\begin{array}{l}\text { epithelial downgrowth G, inflammatory G (contraction of inflammatory membranes and precipitates, Fuchs } \\
\text { heterochromic cyclitis), and neovascular G;* }\end{array}$ \\
\hline - Push I G & postoperative malignant G, uveal effusion syndrome; \\
\hline - Push 2 G & $\begin{array}{l}\text { endophytic tumors, tight encircling procedure, excessive gas or silicone oil intravitreal injection in retinal } \\
\text { detachment surgery, hemorrhage within the suprachoroidal, and supraciliary space; }\end{array}$ \\
\hline - TRAG & the cases in which TR is altered by an identifiable cause. \\
\hline
\end{tabular}

Notes: *The neovascular G has 2 subgroups: type I neovascular G ("organic"), more frequent, in which the retinal ischemia is secondary to vascular alterations (diabetic, atherosclerotic, obstructive etc); and type 2 neovascular G, ("functional"), in which the retinal ischemia is secondary to the compression of relatively normal vessels by the chronic high IOP itself during the terminal phase of any type of pressure aggression $\mathrm{G}$. 
Table 3 The repartition of clinical entities in the pathogenic forms of perinatal G

\begin{tabular}{|c|c|}
\hline Pathogenic form & Clinical entities \\
\hline \multicolumn{2}{|c|}{ Primary perinatal G } \\
\hline \multirow[t]{2}{*}{ - Trabecular G } & trabeculo-schlemmal dysgenesis or agenesis, being merely a histopathologic diagnostic after the \\
\hline & postoperative examination of the trabeculectomy strip; \\
\hline - Pupillary G & G in microphthalmia, microcorneea, microspherophakia; \\
\hline - Angular G & G with Barkan's membrane; \\
\hline - Push G & G in persistent hyperplastic primary vitreous. \\
\hline \multicolumn{2}{|c|}{ Secondary perinatal G } \\
\hline - Exogenous G & $\begin{array}{l}\text { in Sturge Weber syndrome, severe alkali burns, cavernous or dural arterio-venous shunts, orbital disease } \\
\text { that blocks the venous circulation of the orbit; }\end{array}$ \\
\hline - Trabecular G & phakolytic, phako-anaphylactic, uveitic with open angle, tumoral; \\
\hline - Pupillary G & phakomorphic, phakotopic, uveitic with pupil seclusion-occlusion; \\
\hline - Angular G & $\begin{array}{l}\text { neovascular } \mathrm{G} \text { produced by angiogenic factor release from the ischemic retina (in retinopathy of } \\
\text { prematurity, retinoblastoma, medulloepithelioma, familial exudative vitreoretinopathy, Coats' disease, Old } \\
\text { retinal detachment); }\end{array}$ \\
\hline - Push I G & postoperative malignant G; \\
\hline - Push 2 G & endophytic tumors. \\
\hline
\end{tabular}

two forms showing both clinical and therapeutic differences, I named the common type "type 1 (organic) neovascular G," because this form is caused by organic vascular alterations, and is more frequent. I named the form in which the retinal ischemia is secondary to the compression of relatively normal vessels by chronic high IOP "type 2 (functional) neovascular $\mathrm{G}$," because it is caused by the functional impairment of blood vessels, disappears shortly after the vessels are no longer compressed by the high IOP, and is less frequent.

In perinatal $\mathrm{G}$, be it a unique manifestation or a part of a syndrome, the locus in the scheme is determined by its pathogenic mechanism. Because of case rareness, the pathogenic knowledge is limited, so that the distribution of different clinical entities in the pathogenic forms of perinatal $\mathrm{G}$ may represent merely suppositions (Table 3 ).

As for the mixed forms of both perinatal and adult $\mathrm{G}$, their pathogenesis may associate primary mechanisms only, both primary and secondary mechanisms, or secondary mechanisms only. This complex combination accounts for the wide interindividual variability in clinical manifestation of $\mathrm{G}$.

\section{Discussion}

The Discussion section here respects the order in which the issues stirred by the 2008 EGSc were analyzed in my first paper. $^{3}$ To avoid typographic wasted space, the debatable aspects are not restated herein but are summarized in Table 1. That is why I recommend first searching the discussed aspect in Table 1 before passing to any particular subtopic in the present paper (the position in the table is indicated in the text as the heading of the line: for example line C-I-3-c-x). Then I recommend reading the detailed discussion in my first paper ${ }^{3}$ (the page, column, and paragraph where the discussion can be found in my first paper are indicated in Table 1, at the end of each line in the outline in parentheses). Armed with this refreshed information, the reader will easily understand the way in which my classification resolves each discussed aspect.

The suggested classification exceeds the only merit of 2008 EGSc (Table 1, line A) of using the pathogenic criterion for the differentiation of the acquired secondary forms. In my classification, the pathogenic criterion is used as the fundamental criterion valid for all forms. If this classification will accepted, its users would be forced to pursue the thinking pathway up to the clarification of every pathogenic aspect, whether present or potentially dangerous in the future. Thus, the confusions leading to therapeutic disasters will have less chance to appear.

The suggested classification annuls the two steps behind found in the 2008 EGSc, as compared with other existing classifications (Table 1, lines B1 and B2): the etiologic and the gonioscopic criteria - abandoned by the 2008 EGSc for the congenital $\mathrm{G}$ - are used in my classification as secondary criteria, valid for all pathogenic forms.

The suggested classification annuls the shortcomings of any existing classification of glaucomas, the 2008 EGSc included (Table 1, lines C-I through C-IX).

\section{The suggested classification does not use criticizable fundamental criteria}

The first criticizable fundamental criterion in all previous classifications is the genetic criterion (Table 1, line C-I-1). My classification does not use it anymore because the congenital-acquired dichotomy is no longer necessary, 
both from the conceptual and from the practical points of view. From the conceptual point of view (Table 1, line C-I-1-a), the suggested classification is built on the idea that the iridocorneal angle configuration and the trabecular meshwork alteration (characteristics that define the primary forms of adult acquired $\mathrm{G}$ in any existing classification) are anatomic or functional anomalies with congenital origin and very late pathologic manifestation. If most primary forms of adult acquired $\mathrm{G}$ have congenital origins, while some congenital $\mathrm{G}$ forms are acquired (secondary to a cause), then the congenital-acquired dichotomy cannot clearly separate the cases and loses its reason to exist. In my classification, it has been perfectly replaced by the primary-secondary dichotomy: the primary cases have congenital origins (and early, late, or very late clinical manifestations), while the secondary cases are acquired.

The congenital-acquired dichotomy is also not necessary from the practical point of view (Table 1, line C-I-1-b): it conveys erroneous information about the age when the IOP begins to increase, information with important clinical and therapeutic impact. In all existing classifications, the term "congenital" has birth-related connotations. Nevertheless, the clinical behavior leading to a specific therapeutic approach depends more on an IOP increase before 2 years of age than on the presence of causal angle anomalies at birth. As a consequence, there is no need to retain birth as the universal landmark. I only suggest moving this landmark to 2 years of age and replacing the term "congenital" with "perinatal." With this term, the difficulties encountered in congenital G classification have disappeared: the "perinatal-adult" dichotomy conveys the necessary information referring to age only and eliminates the unwanted genetic and etiologic connotations implied by the "congenital-acquired" dichotomy.

My classification does not use the etiologic and gonioscopic criteria as fundamental ones, and the shortcomings listed in Table 1 as lines C-I-2 and C-I-3-a no longer exist.

As the gonioscopic criterion has ceased to be fundamental, my classification is able to cover all cases, resolving thus the shortcoming listed as line C-I-3-b in Table 1: the pathogenic mechanism of TRAG, exogenous G, and neovascular $G$ have no connection with the preexistent angle configuration. These forms are classified according to other criteria at completely different levels than those separated by gonioscopy.

Some statements with gonioscopic tint in Terminology and Guidelines for Glaucoma ${ }^{1}$ (Table 1, line C-I-3-c), may raise serious doubts regarding the validity of 2008 EGSc and may favor its abandonment. First, the users might not understand why an angle closed for $90^{\circ}$ (a quadrant from its length) is considered open (Table 1, line C-I-3-c-x). This doubt will no longer exist if, instead of insisting on a certain degree of angle openness, pathogenic mechanisms are considered. Gonioscopic terms like OAG and ACG may lead one to search in two opposite directions, which may result in not finding the correct treatment because different mechanisms may produce the same degree of angle openness, whereas the same mechanism may act on different degrees of angle openness. On the contrary, when one uses pathogenic terms, the mechanism is already clarified, and the doctor knows what must be done to resolve the case. The correct attitude is not dictated by the $10^{\circ}$ that separates $85^{\circ}$ from $95^{\circ}$ but by the case analysis in its whole complexity, taking into consideration the presence of ocular and familial aggravating factors (evolutive cataract, extensive posterior synechia, history of angle closure in the other eye or among blood relatives) and the aspect in the rest of the angle. If the angle is narrow all around and closed for $85^{\circ}$, after excluding other forms of pretrabecular $\mathrm{G}$, it is safer to consider the case as pupillary $\mathrm{G}$ in precrisis or intercrisis and act accordingly before major decompensation occurs. If the rest of the angle is widely open, one must search for the cause of posterior push and act accordingly. In my opinion, the difference between $85^{\circ}$ and $95^{\circ}$ is inconsistent.

The second statement (Table 1, line C-I-3-c-y) suggests that the correct formulation of diagnostic terms according to the 2008 EGSc cannot always help the therapeutic choice. After arriving at the end of the thinking pathway imposed by this classification and after spending enough time to place a new case in the group of ACG or OAG, one may expect to receive help in therapeutic decision making. Instead, one discovers that the same mechanism may lead both to OAG and ACG. Knowing that the treatment must attack the pathogenic chain, one may ask: what was the use of the whole previous effort? To prevent abandonment, a good classification must have practical finality and must help the doctor to conduct the treatment. As the same gonioscopic form may be produced by different pathogenic mechanisms, and as the specific treatment is addressed to the mechanism, the best solution is to conduct the treatment not after a clinical sign (the gonioscopic aspect) but after the pathogenic mechanism that has produced that particular sign. It is easier to apply pathogenic thinking when guided by a pathogenic classification than by any of the existing classifications. After framing the case in one category, these classifications leave the doctors in the middle of nowhere and force them to a new level of analysis, this time a pathogenic one, before being 
able to understand the case and find the correct treatment. Contrary to what happens when using these classifications, after reaching the end of the thinking pathway imposed by my classification, doctors will know both the exact location of their patients on the sickness map and what to do to bring them home. Once the users see things more clearly, the tendency to abandon the suggested classification will become less frequent.

A third reason for abandonment is represented by the fact that at least one attitude recommended by the 2008 EGSc (Table 1, line C-I-3-c-z) has not prevented the visual loss: too many frank attacks of angle closure (AC) have appeared because the Terminology and Guidelines for Glaucoma has excluded the prophylactic treatment in cases showing iridotrabecular contact for less than $180^{\circ}$. As far as I am concerned, I am convinced that without successful treatment, an angle closure of less than two quadrants may evolve sooner or later into a clinically manifested closure. If the angle is narrow all around and closed for as little as $10^{\circ}$, if the already mentioned aggravating factors exist, and if other causes of pretrabecular $\mathrm{G}$ have been excluded, it is safer both for the doctor and for the patient to consider the case as "pupillary G in precrisis or intercrisis" and to practice peripheral iridectomy (PI) even if the provocative tests remain negative for the moment. This attitude is safe because angle closure may occur sooner or later, whereas there is no certainty that the patient will immediately present himself to the doctor. For such patients, it is better to consider that the category "primary angle closure suspect" does not exist. I believe that it is unwise to delay the PI on the basis of the risks and complications the procedure implies; the risks are practically annulled, and the rare complications are easily resolved by modern surgery. If we accept the idea of implanting an intraocular contact lens (although nothing forces us to practice this cataractogenic procedure), why postpone the PI when the risk of $\mathrm{AC}$ is certain and may produce blindness, and cataract surgery will become necessary in a few years anyway? With such patients in such environmental conditions, it is unwise to remain confined to the 2008 EGSc project and refuse the PI based on nonexistent reasons. It is time to adapt our attitude to 21 st century exigency that no patient should lose his sight because of neglected pupillary G.

\section{The suggested classification does not use criticizable secondary criteria}

The first criticizable secondary criterion in 2008 EGSc is the use of the association with other congenital anomalies as the first secondary criterion (Table 1, line C-II-1). In my previous paper, ${ }^{3}$ I proved that its use at such an important crossing is illogical and nonproductive: it cannot clarify the ocular hypertension mechanism nor facilitate the therapeutic decision making. In my classification, the information offered by general examination (the association with other congenital anomalies) is used for each pathogenic form, but only at the end, when each clinical manifestation must find its locus.

Secondly, when the 2008 EGSc discusses pathophysiologic mechanisms, it mixes them with blocking agents (Table 1, line C-II-2). In the suggested pathogenic classification, when we speak about pathophysiologic mechanisms, we mean pathophysiologic mechanisms. The blocking agents are discussed later, when the clinical forms are differentiated.

Thirdly, the 2008 EGSc uses the clinical stages for the differentiation of PACG (Table 1, line C-II-3). This is a criticizable manner of using this criterion as the first secondary one. More important would have been the further differentiation of PACG according to the pathogenic mechanism (pupillary $\mathrm{G}$, angular $\mathrm{G}$, push $\mathrm{G}$ ), because significant differences in therapeutic attitude between these pathogenic forms exist, and are not emphasized in any way by the clinical staging. Moreover, not every form of PACG passes through the listed stages (for example, the primary malignant $\mathrm{G}$, form of PACG). I think that it is not recommendable to describe the clinical stages as valid for the whole pathogenic group when not all its members will pass through these stages.

In my classification, the clinical stages are used, but only at the end, after the identification of each clinical entity that enters each pathogenic form. At this point of the discussion, one must remember that: 1) some primary pupillary and angular Gs, may pass through the stages listed in Terminology and Guidelines for Glaucoma ${ }^{1}$ (precrisis, attack, postattack, intercrisis, and chronic); 2) these stages are valid for some secondary Gs, too; 3 ) in other forms of secondary $\mathrm{G}$ other stages should be used (for example, in neovascular G, only 2 clinical stages exist: OA neovascular $\mathrm{G}$, which is reversible by angiogenesis factor annulment, and AC neovascular $\mathrm{G}$, which is irreversible, because the angle is closed by strong anterior goniosynechiae that need to be solved by G surgery); 4) when the neovascular $\mathrm{G}$ is analyzed, one must not repeat the 2008 EGSc error of forgetting the terminal stage of neovascular $G$ that may develop in any form of block $\mathrm{G}$ manifested by chronic high levels of IOP.

Finally, the pathogenic criterion is used as the fundamental criterion, valid for all forms of $\mathrm{G}$, so that the shortcomings C-II-4 and C-IV-5 in Table 1 no longer exist. 


\section{For each crossing, the suggested classification uses only one criterion}

Thus, the shortcomings listed under Table 1, line C-III have been eliminated. The result is a better understanding of the sickness, both in general and in any particular case a doctor has to resolve. The tendency to abandon this classification will no longer appear.

\section{The suggested classification uses each criterion in a consistent manner}

Unlike the 2008 EGSc, which uses three fundamental criteria at the first crossing, my classification uses only one, but maintains it for the first five to seven successive crossings, until its differentiation capabilities are exhausted. After that, the secondary criteria pick up the relay, until each form finds its natural place.

The signs of inconsistency observed in 2008 EGSc are resolved as follows: 1) the gonioscopic and etiologic criteria are used as secondary criteria for all cases (Table 1, line C-IV-1); 2) the association with other congenital anomalies is used but only at the end, together with other clinical aspects, when a locus must be created for each clinical entity (Table 1, line C-IV-2);3) age has been advanced to the rank of second secondary criterion, replacing the genetic one and allowing the separation of two groups with clinical and therapeutic significance: the perinatal-adult dichotomy resolves any unclear aspects found in the classic category of congenital $\mathrm{G}$ (Table 1, line C-IV-3); 4) the clinical forms of perinatal $\mathrm{G}$ are distributed into the same pathogenic categories as the ones used for adult G (Table 1, line C-IV-4). Nevertheless, we must admit that the distribution suggested in this paper is only a first attempt that needs further investigation and debate.

With all these improvements, confusion is less likely to occur.

\section{The suggested classification respects the reality}

The mechanism of TRA is no longer exclusively connected with open angle and low pressure (Table 1, lines C-V-1 and $\mathrm{C}-\mathrm{V}-2)$. We are allowed to think of neuroprotection in any form of G, not only in OAG but also in ACG, not only in primary but also in secondary $\mathrm{G}$, not only in normotensive but also in hypertensive cases, and not only in adults but also in infants. In perinatal $\mathrm{G}$, the tissue resistance acts not only at the level of optic neural tissue, but also at the level of sclera. At this level, it has a positive role, the parietal distension delaying in some measure the effects of IOP increase on the neural tissue.
The mechanism of episcleral vein pressure increase (Table 1, line C-V-3) is no longer exclusively connected with open angle. We are allowed to consider that the exogenous $\mathrm{G}$ may appear at any degree of angle aperture and that angle closure may appear in a case with exogenous $\mathrm{G}$.

The neovascular G (Table 1, line C-V-4) is a "secondary angular G," and no logic fracture will appear when we will describe two evolutive stages using the gonioscopy as one of the third-rank secondary criteria: OA neovascular $\mathrm{G}$ (reversible) and $\mathrm{AC}$ neovascular $\mathrm{G}$ (irreversible), each with specific treatment.

The plateau iris $\mathrm{G}$ (Table 1, line C-V-5) is a "primary angular G." The great advantage of the term "angular G" is that it allows the use of the pathogenic criterion both in primary and secondary forms, thus avoiding the shortcomings connected with the OAG-ACG dichotomy.

\section{The new classification frames each clinical entity in one pathogenic locus}

The fact that after the pathogenic mechanism elucidation, the doctor will no longer be forced to place the case among either congenital or acquired forms, will annul the intellectual turmoil (Table 1, line C-VI) caused by the fact that any existing classification frames many clinical entities in both congenital and acquired forms - the two main, opposite, and mutually exclusive categories separated at the first crossing. The perinatal-adult dichotomy is logical and more permissive. Its use will not stir debate, because it does not combine genetic with etiologic connotations, such as those found in the congenital-acquired dichotomy. It refers only to age, and it is not against logic to accept that one sickness may appear in infants as well as in adults.

The clinical entities listed in the corresponding section of my first paper ${ }^{3}$ as being included in more than one category by the 2008 EGSc will be classified according to the pathogenic mechanism in one of the pathogenic forms. Then, terms like "primary" or "secondary" will indicate the absence or presence of an identifiable cause. Thereafter, the term "perinatal" will indicate the appearance before 2 years of age, the age at which buphthalmia is usually associated. When more than one mechanism contributes to the pressure increase, the case may enter the "mixed perinatal G" group. When the mechanism is unclear at the moment, the entity may enter the "uncertain or unknown pathogeny perinatal G" group: such inclusion has the great advantage of bringing the forms with unclear pathogeny to the researcher's attention instead of being indistinctly forgotten among congenital Gs, where they have been left undisturbed over the last 70 years. 


\section{The suggested classification allows the framing of all forms of sickness}

The pathogenic diagnostic term for the first case (Table 1, line C-VII-1) is "trabecular on narrow angle G." The term indicates both the main mechanism and the risk of $\mathrm{AC}$, which may affect the patient or his blood relatives. At the same time, the term avoids the confusion generated by the contradictory definitions suggested by the 2008 EGSc.

The pathogenic diagnostic term for the second case (Table 1, line C-VII-2) is "primary trabecular and pupillary $\mathrm{G}$, in attack, left eye and in precrisis, right eye". If cataract contributes to the condition, the case is denoted as "primary trabecular and secondary phacomorphic pupillary G." Thus, avoiding the terminological confusion implied by terms like "POAG and AC," the doctor will receive complete information so as to adopt the correct therapeutic decision for both the patient and his blood relatives. More importantly, the doctor will do this as soon as he or she reads the diagnostic term.

Similarly, the third case (Table 1, line C-VII-3) is "primary pupillary and secondary trabecular G," while the three cases in Table 1, line C-VII-4 are "primary trabecular G with TRA," "primary trabecular G," and "primary trabecular G with TRI (tissue resistance increase)." Reading such diagnostic terms, the doctor will automatically recall all considerations detailed in the corresponding paragraph of my previous paper. Naturally, he or she will reach the stage of therapeutic decision making sooner; more importantly, the decision will be correct.

The two cases in Table 1, line C-VII-5 are "primary pupillary G with TRA," and "primary pupillary G with TRI." The information received by the doctor is much more complete, and the therapeutic attitude will be correct, affecting both the patient and his blood relatives.

Finally, the pathogenic diagnostic terms of the last cases in Table 1, line C-VII-6 are, in order: "primary exogenous and secondary phacomorphic pupillary G," "primary chronic pupillary and secondary exogenous G," "primary trabecular and pupillary G," and "primary tissue resistance alteration G with primary trabecular G."

\section{The new classification offers direct therapeutic suggestions}

In simple pathogeny $\mathrm{G}$, a specific treatment exists for each pathogenic group.

\section{The primary forms}

The pathogenic treatment in case of trabecular $\mathrm{G}$ aims for outflow increase: in the reversible stage of the sickness, the treatment is medical. In the irreversible stage, the treatment is surgical: the first surgery is perforating (with or without trabecular bypass), or nonperforating (trabectomy included); in case of relapses, the surgery must associate fibrogenesis inhibitors and/or modulators; when all these procedures fail, one must try glaucoma drainage devices, as the last resource before cycloanemisation.

The pathogenic treatment in case of pupillary $\mathrm{G}$ consists in by-passing the pupillary block. In the reversible stage, the treatment is peripheral iridectomy (PI) from the first moment.

In the irreversible stage, after long-standing angle closure (3-10 days), a new phenomenon superimposes, one that cannot be solved by PI: the persistent iris deformation and the associated inflammation may produce fibrous membranes that enwrap or penetrate the iris. These structures tend to recreate the iris deformation with anterior contact and anterior synechia relapse, in spite of the fact that the PI has equalized the pressures in front and behind the membrane.

I named this manifestation "iris vicious position memory, $" 23,24$ a manifestation that may also appear in neglected perforant corneal wounds with iris hernia. In such neglected cases, after resolving the iris block by PI, we must annul the iris vicious position memory by using goniosynechialysis, ${ }^{25}$ or better, angle repermeation. ${ }^{26}$ The goniosynechialysis separates the synechia under gonioscopic control either by passing a spatula ${ }^{25}$ or by injecting cohesive OVD. ${ }^{27} \mathrm{I}$ believe that the failure rate of up to $58 \%{ }^{28}$ is caused by the fact that the fibrous structures that enwrap or penetrate the iris are not disturbed by this procedure and recreate the iris deformation, with corneal contact and synechia relapse, in spite of a large PI.

Angle repermeation ${ }^{26}$ produces better results because after PI, the angle is opened by centripetal tractions on the pupil margin, up to $1 \mathrm{~mm}$ from the pupil center, in the zone of the anterior synechiae. This maneuver tears both the synechiae and the fibrous structures that are responsible for iris vicious position memory and for synechiae relapse. In the great majority of cases, the IOP returns to normal or may remain for 2 to 4 days at around $30 \mathrm{mmHg}$ with maximal medication. After this short period of trabecular structure rearrangement, the IOP usually decreases, allowing progressive tapering of medication. In the end, the case may remain compensated for life, usually with but also without medication. The results after angle repermeation, ${ }^{29}$ are shown in a new manner of presentation ${ }^{30}$ that conveys all necessary data for statistical interpretation at five significant evolution moments: 


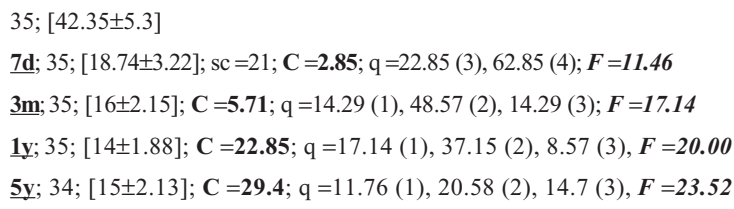

In the first line of this formulation, the figures represent: the number of cases that entered the study; and the IOP [expressed as mean \pm standard deviation $(\mathrm{m} \pm \mathrm{SD})$ ]. In the other lines, there exists a column for the time intervals to the postoperative control ( $\mathbf{d}=$ days, $\mathbf{m}=$ months, $\mathbf{y}=$ years); a column for the number of patients that passed each control; a column for IOPs [expressed as $\mathrm{m} \pm \mathrm{SD}$ ]; a column for the complete success rates $(\mathbf{C})$; a column for the qualified success rates (q), detailed according to the number of medications that ensured the compensation (between parenthesis); and a column for failure rates $(\boldsymbol{F})$, in order to ease the understanding of the success-failure rapport. Moreover, the small indent in the complete success column (at the second line) highlights the success criterion ( $\mathrm{sc}=21$ : expressed in $\mathrm{mmHg}$, as the superior limit; sc $=30 \%$ : expressed as percentage of IOP reduction; $\mathrm{sc}=\mathrm{tp}$ : expressed as target pressure; $\mathrm{sc}=\mathrm{np}$ : expressed as normative pressure).

This reporting manner was designed to replace in $G$ abstracts the "complete - qualified" success dichotomy, because the formulation in words of the results takes with accompanying phrasing more typographic space, in spite of the fact it does not transmit anything about the preoperative aspect, about the success criterion, about the number of cases that passed each significant control, about the IOP mean value and SD at each control, about the number of medications that ensured the qualified success at each control. In a world based on an extensive use of abstracts for reviewing information, the suggested manner of reporting results would bring satisfaction to all areas of the process: publishers would save typographic space, readers would find all the necessary data for statistical analysis and comparison with other studies, and authors would be convinced that the essence of their work would penetrate in spite of any economic, linguistic, or political barriers.

The results after angle repermeation presented in the formula above prove that the neglected pupillary $\mathrm{G}$ may be successfully treated without trabecular surgery. For those who have noticed, the increase in complete success rate after 3 months is explained by the fact that cataract surgery with IOL implantation was practiced in $68.57 \%$ of cases between 3 months and 5 years, anytime the light projection was recovered in at least 2 quadrants). The well known positive effect of cataract surgery on ACG does not change my message: the neglected pupillary G may be successfully treated without trabeculectomy.

Based on these results, I think that, contrary to what happens in many parts of the world, the trabeculectomy should no longer be practiced as surgery of first intention in any neglected case of pupillary $\mathrm{G}$ by anyone using the pathogenic thinking pathway. If in such cases one uses trabeculectomy before attempting angle repermeation, one frequently leaves a perfectly functional trabeculum buried under the iris block. One imposes the trabeculectomy risks and complications on patients who could have their glaucoma resolved without these risks and by using a much shorter procedure. Taking all these arguments into consideration, the only logical conclusion is that the trabecular surgery should be reserved only for trabecular $G$ or for the forms of mixed $G$ in which the trabecular block is associated. In case of neglected pupillary $\mathrm{G}$, trabecular surgery should be practiced only as a surgery of second intention, when the failure of PI + angle repermeation + tolerable medication has proven that an important trabecular alteration exists and is unresponsive to medication. In my 20 years of experience with angle repermeation, the residual hypertonia of $30 \mathrm{mmHg}$ with maximal medication 5 days after surgery is the first indication for trabecular surgery. In the first 3 postoperative months, any durable IOP rise above $21 \mathrm{mmHg}$ with tolerable medication (or above the target pressure established according to the stage of sickness) represents a similar indication. That is why frequent controls are needed in the first 3 postoperative months (day 1, 5, 15, $30,45,60,90)$. Generally, the results at 3 months are long duration ones.

After closing this long discussion about the pathogenic treatment in case of pupillary $\mathrm{G}$, the other forms need less discussions.

In the case of the adult form of primary angular G (plateau iris) the treatment of the reversible stage is to deepen the angle working on the iris side (flattening of the last iris fold by argon laser iridoplasty), with or without medical therapy. In the irreversible stage, the treatment is to deepen the angle toward the exterior by trabeculectomy, with or without medical therapy. In such cases, the nonperforant filtering procedures cannot prevent AC.

In the case of perinatal angular $G$, the treatment is goniotomy or trabeculotomy, with or without medical therapy.

The primary forms of push $\mathrm{G}$ may exist only as push $1 \mathrm{G}$ (functional). In the reversible stage of both malignant $G$ and of uveal effusion syndrome, the treatment is atropine and anti-inflammatory agents as well as complex antiglaucoma 
medication, except miotic drugs. In the irreversible stage of malignant $\mathrm{G}$, if UBM reveals supraciliary accumulation, the treatment is a scleral puncture (which may be blocked, with relapse) or, better, a filtering puncture (a 2/2 mm angulated incision with the excision of the flap tip), ${ }^{31}$ which in my hands has never failed. When the aqueous humor is misdirected toward a deep intravitreal or retrovitreal space, the treatment is puncture in pars planum and vitreal aspiration, which is supposed to create double hyaloidotomy. In case of relapse, when the dense vitreous rapidly closes the tunnel, the treatment is lensectomy and vitrectomy, the purpose of which is to create wide communication between the anterior chamber and the intraretrovitreal space through the pupil. In case of uveal effusion not responding to medical therapy, the treatment is the Gass procedure ${ }^{32}$ or filtering punctures practiced at the equator. ${ }^{31}$

\section{The secondary forms}

In the reversible stage, the treatment is medical treatment of $\mathrm{G}$ as well as specific treatment of the identifiable cause. In the irreversible stage, the treatment is specific treatment of the identifiable cause in addition to surgery using the protocol of the primary form with or without medical therapy.

Some secondary forms deserve special mention.

In the case of exogenous $\mathrm{G}$, the pathogenic treatment aims for inflow reduction: by medical means in its reversible stage (carbonic anhydrase inhibitors, even apraclonidine, because of its vasoconstrictive effects on ocular and orbital arterioles ${ }^{33}$ ), and by surgical means in its irreversible stage (cycloanemisation, practiced with prudence, because if excessive, it may result in an atrophic eye). In case of idiopathic elevated episcleral vein pressure, the perforant filtration surgery (or, better, the nonperforant one) may have good results, although it is frequently complicated by choroidal effusion or hemorrhage.

In neovascular $\mathrm{G}$, a form of secondary angular $\mathrm{G}$, the following treatments can be suggested. In the case of type 1 neovascular $\mathrm{G}$ (organic), and its $\mathrm{OA}$, reversible phase, the treatment is to inhibit the vasoformative factor, by laser or cryogenic ablation of peripheral retina, or by intravitreal injection of an anti-VEGF agent: these procedures can cure the neovascular $\mathrm{G}$ itself. In the $\mathrm{AC}$, irreversible phase of this type of neovascular G, the treatment is trabeculectomy, but only after drying the neovessels. (If one attempts to practice trabeculectomy before drying the neovessels, the result will be a profuse, relapsing hyphema that will block the newly created outflow pathway, with failure and hematic impregnation of the cornea). On the contrary, in the case of type 2 neovascular $\mathrm{G}$ (functional), produced by the chronic high IOP itself, the causal $G$ has already reached its irreversible phase, so that the G surgery is compulsory. The essential difference as compared with the neovascular type $1 \mathrm{G}$ consists in the fact that there is no need to dry the neovessels before G surgery: if successful, the $\mathrm{G}$ surgery will annul the retinal ischemia, the cause of the vasoformative factor. After a few weeks of relapsing hyphema with normal IOP, this attitude will cure both the glaucoma and its neovascular complication.

In secondary push $1 \mathrm{G}$ (malignant), beside the protocol used in primary forms, laser hyaloidotomy may be effective on aphakic or pseudophakic eyes. In secondary push 2 Gs (organic), each causal type responds to a specific treatment. For tumors, the treatment would be ablation of the tumor (using chemical, radiation, or surgical means) or of the eye. For excessive maneuvers in retinal detachment surgery, the treatment would be the release of the encircling suture or partial evacuation of the intravitreal content; for blood (expulsive hemorrhage), the treatment would be drainage by simple puncture (which is blind, risks aggravating the hemorrhage, and may be blocked, with hypertonia relapse) or by Z-shaped wide sclerotomy placed under horizontal recti muscles. ${ }^{34}$ This procedure searches the blood collection, drains it selectively, and - in my experience on six cases has never failed.

In case of complex pathogeny $\mathrm{G}$, the doctor will borrow from the treatment of each simple pathogeny form as much as is needed to cover every pathogenic aspect of the case.

\section{The suggested classification avoids the shortcomings listed as miscellanea}

The absence of a definition and the terminologic confusions listed in Table 1, lines C-IX-1 and C-IX-2-a have been resolved by the replacement of the congenital-acquired dichotomy with the perinatal-adult one. The last two sources of confusion (Table 1, lines C-IX-2-b and C-IX-2-c) no longer exist, because my classification recognizes only three mechanisms of pretrabecular block: pupillary block, angular block, and the posterior push. These mechanisms are active in both primary and secondary, as well as in perinatal and adult forms. As a consequence, the torrent of questions at the end of my previous paper ${ }^{3}$ remains without object.

Summarizing the discussion, the suggested classification fulfills all the conditions of a good classification listed in the introductory lines of this study: it is comprehensive, clear, and open, it helps in understanding the sickness and eases the decision making. In this classification, everything 
is clear from the beginning to the end. The doctor, both the experienced and the inexperienced, will know which mechanisms must be therapeutically addressed and which are the traps and pitfalls that can lead to error. Experienced doctors will no longer have to think: "The 2008 EGSc says this, but I know that the reality is different." Finally, the researchers will have the forms that need further investigation gathered in the category of unknown or uncertain pathogeny $\mathrm{G}$.

Looking at the subject from this new perspective, one may easily see that the new classification does not require major changes after all. Naturally, the categories have received new pathogenic names, but the clinical forms in each category remain mostly the same. Some categories have been upgraded and received the right of independent existence (TRAG, exogenous $\mathrm{G}$ ) but only because their natural place was not among POAGs. Although the scheme seems more complex, one must consider that for the great majority of patients, one will use only the final fifth or sixth crossings, which in fact reflect the gonioscopic classification. All one needs to know is that 1) more mechanisms may act on the same case, 2) exogenous $\mathrm{G}$ may appear on any degree of angle aperture, and 3) somewhere, at a higher level, TRA exists and may complicate the evolution of any gonioscopic form, primary or secondary. To reflect this reality, the exogenous $\mathrm{G}$ and the TRAG have received the right of independent existence, the mixed forms have been included in the scheme, and a locus has been created for cases with unknown or uncertain pathogeny.

On the other hand, if one examines the benefits, one cannot deny the fact that although the scheme seems more complex, it eases the decision making. Once the pathogenic mechanism is determined, my classification suggests the pathogenic treatment, which must be immediately initiated. This pathogenic treatment offers time for etiologic analysis, because in secondary forms, an etiologic treatment must attack the cause in parallel with antiglaucoma therapy. In the end, the clinical analysis will accurately identify the clinical form, warning the doctor about particular evolution modalities or about particular responses to the pathogenic or to the etiologic treatment. With such classification having high practical finality and avoiding therapeutic disasters, the users will not show abandonment tendencies, like the one exemplified at page 280 of my previous paper. ${ }^{3}$

As I wrote 22 years ago, ${ }^{9}$ if every doctor stands to benefit from the use of the suggested classification, only tradition and the comfort brought about by habit may justify the further use of gonioscopic classification, which only suggests the mechanism and exposes the patient to therapeutic errors, instead of the pathogenic classification, which clearly defines the mechanism, indicates the correct treatment, and avoids therapeutic errors.

\section{Conclusion}

The suggested classification is a pathogenic, etiologic, and clinical classification that fulfills all the conditions of a good classification. It corrects the drawbacks of all previous classifications. The suggested classification is the first in which the main criterion is constantly used for the first five to seven crossings until its differentiation capabilities are exhausted. Then, secondary criteria pick up the relay until each form finds its logical place in the scheme. To avoid unclear aspects, the genetic criterion is no longer used, having been replaced by one of the clinical criteria (age). The suggested classification brings only benefits to all categories of ophthalmologists: the beginners will have a tool to better understand the sickness and to ease their decision making, whereas the experienced doctors will have their practice simplified. For all doctors, errors leading to therapeutic disasters will be less likely to occur. Finally, researchers will have the object of their work gathered in the group of glaucoma with unknown or uncertain pathogenesis, while the result of their work will easily find a logical place in the scheme, because the suggested classification remains open to any new to development.

\section{Disclosure}

The author reports no conflicts of interest in this work.

\section{References}

1. European Glaucoma Society. Terminology and Guidelines for Glaucoma. 3rd ed. Savona: Editrice Dogma; 2008:93-113.

2. Donders FC. see Hoffmanns: vGraeffes Arch Ophthal. 8(2):124.

3. Bordeianu CD. Critical analysis of the classification of glaucomas issued by the European Glaucoma Society in 2008. Clin Ophthalmol. 2014;8:271-282.

4. Allingham RR, Damji KF, Freedman SF, Moroi SE, Rhee DJ, editors. Shields Textbook of Glaucoma. 6th ed. Philadelphia: Wolters Kluwer/ Lippincott, Williams and Wilkins; 2011.

5. Ourgaud A, Étienne R. L'exploration fonctionnelle de l'oeil glaucomateux. Rapport de la SFO [The functional investigation of glaucoma eye. Report of the French Society of Ophthalmology]. Paris: Masson; 1961:French.

6. Krasnov MM. [Pathogenic forms of glaucoma and principles governing their treatment]. Vestn Oftalmol. 1965;78(5):29-Russian.

7. Goldmann H. Abflussdruck, Minutenvolumen und Wiederstand der Kammerwasserströmung des Menschen [Outflow pressure, minute volume and resistance of aqueous humor flow in man]. Doc Ophthalmol. 5-6:278-356. German.

8. Bordeianu CD. Clasificarea patogenică a glaucoamelor [The pathogenic classification of glaucomas]. Presented at the Monthly Meeting of the Society of Medical Sciences (SSM), Bucharest branch, Romania, January 18, 1986. Romanian.

9. Bordeianu CD. The pathogenic classification of glaucomas. Oftalmologia. 1992;36:331-342. 
10. Bordeianu CD. The gonioscopic classification versus a pathogenic one: past and future in glaucoma classification. Abstracts of the 12th Congress of the European Society of Ophthalmology; June 27-July Stockholm, Sweden. Page 91.

11. Schacknow NP, Samples RJ. The Glaucoma Book: A Practical, Evidence-Based Approach to Patient Care. New York: Springer; 2010.

12. Yanoff M, Duker JS. Ophthalmology. 2nd ed. St Louis: Mosby; 2004.

13. Etter JR, Affel EL, Rhee DJ. High prevalence of plateau iris configuration in family members of patients with plateau iris syndrome. J Glaucoma. 2006;15(5):394-398.

14. Online Mendelian Inheritance in $\operatorname{Man}^{\circledR}\left(\mathrm{OMIM}^{\circledR}\right)$. Glaucoma, normal tension, susceptibility to. \#Baltimore: Johns Hopkins University; [updated August 2010]. Available from: http://omim.org/entry/606657? search=6 06657\&highlight=Accessed June 2014.

15. Hitchings R. Normal tension glaucoma. In: Yanoff M, Duker JS, editors. Ophthalmology. 2nd ed. St Louis: Mosby; 2004:1488-1489.

16. Online Mendelian Inheritance in $\operatorname{Man}^{\circledR}\left(\mathrm{OMIM}^{\circledR}\right)$. Glaucoma, primary open angle; POAG. \#Baltimore: Johns Hopkins University; [updated June 2013]. Available from: http://omim.org/entry/Accessed June 2014.

17. Shaarawi TM, Sherwood MB, Hitchings RA, Crowston JG, editors. Glaucoma. London: Saunders Elsevier; 2009.

18. Alvarado I, Murphy C, Juster R. Trabecular meshwork cellularity in primary open-angle glaucoma and nonglaucomatous normals. Ophthalmology. 1984;91:564-579.

19. Babizhayev MA, Brodskaia MW. Fibronectin detection in drainage outflow system of human eyes in ageing and progression of open-angle glaucoma. Mech Ageing Dev. 1989;47:145-157.

20. Lütjen-Drecoll E, May CA, Polansky JR, Johnson DH, Bloemendal H, Ngyuen TD. Localization of the stress proteins alpha B-crystalline and trabecular meshwork inducible glucocorticoid response protein in normal and glaucomatous trabecular meshwork. Invest Ophthalmol Vis Sci. 1998;39:517-525.

21. Bhattacharya SK, Rockwood EJ, Smith SD, et al. Proteomics reveal Cochlin deposits associated with trabecular meshwork. J Biol Chem. 2005;280:6080-6084.

22. Lütjen-Drecoll E, Shimizu T, Rohrbach M, et al. Quantitative analysis of "plaque material" in the inner and outer wall of Schlemm's canal in normal- and glaucomatous eyes. Exp Eye Res. 1986;42:443-455.
23. Bordeianu CD. A new notion: "the iris vicious position memory." Abstracts of the 12th Congress of the European Society of Ophthalmology; June 27-July Stockholm, Sweden. Poster SPpage 178.

24. Bordeianu CD. Again, about iris vicious position memory. Abstracts of the 5th Congress of the Romanian Society of Ophthalmology; 4-7 October 2006, Sinaia, Romania, page 15.

25. Campbell DG, Vela A. Modern goniosynechialysis for the treatment of synechial angle-closure glaucoma. Ophthalmology. 1984;1052-1060.

26. Bordeianu CD. Angle repermeation, surgery of choice in neglected pupillary glaucomas. Proceedings of the 14th Congress of the European Society of Ophthalmology, Madrid, Spain, June 7-Bologna: Monduzzi Editore. 2003:159-164.

27. Varma D, Baylis O, Wride N, Phelan PS, Fraser SG. Viscogonioplasty: an effective procedure for lowering intraocular pressure in primary angle closure glaucoma. Eye (Lond). 2007;21:472-475.

28. Tanihara H, Nishiwaki K, Nagata M. Surgical results and complications of gniosynechialysis. Graefes Arch Clin Exp Ophthalmol. 1992;230:309-313.

29. Bordeianu CD. Again, about angle repermeation. Abstracts of the 5th Congress of the Romanian Society of Ophthalmology; 4-7 October 2006; Sinaia, Romania. Page 18.

30. Bordeianu CD, Ticu CE. A new manner of reporting pressure results after glaucoma surgery. Clin Ophthalmol. 2012;6:23-31.

31. Bordeianu CD. Punctia filtranta in chirurgia decolarii de coroida [Filtering puncture in choroidal detachment surgery]. Presented at the $21 \mathrm{st}$ Annual Meeting of Ophthalmologists from Romania (RAO), 23-25 May 1985, Piatra Neamt. Romanian.

32. Gass JD. Uveal effusion syndrome: A new hypothesis concerning pathogenesis and technique of surgical treatment. Retina. 1983;3:159-163.

33. Montzioros N, Weinreb RN. Apraclonidine reduces the intraocular pressure in eyes with increased episcleral venous pressure. J Glaucoma. $1992 ; 1: 42-43$

34. Bordeianu CD. L'hémorragie expulsive: accident insurmontable? Discussion d'une possibilité de traitement [Is expulsive hemorrhage an accident insurmountable? Discussion about a possibility of treatment] J Fr Ophthalmol. 1982;5(4):257-281. French.
Clinical Ophthalmology

\section{Publish your work in this journal}

Clinical Ophthalmology is an international, peer-reviewed journal covering all subspecialties within ophthalmology. Key topics include: Optometry; Visual science; Pharmacology and drug therapy in eye diseases; Basic Sciences; Primary and Secondary eye care; Patient Safety and Quality of Care Improvements. This journal is indexed on Submit your manuscript here: http://www.dovepress.com/clinical-ophthalmology-journal

\section{Dovepress}

PubMed Central and CAS, and is the official journal of The Society of Clinical Ophthalmology (SCO). The manuscript management system is completely online and includes a very quick and fair peer-review system, which is all easy to use. Visit http://www.dovepress.com/ testimonials.php to read real quotes from published authors. 